\title{
Investigation on the Characteristics of Water Flow over a Backward Facing Step under High Reynolds Number with Particle Image Velocimetry
}

\author{
Fan Xinjian \\ ${ }^{1}$ Nanjing Hydraulics Research Institute, Nanjing 210029, China \\ ${ }^{2}$ School of Energy and Power Engineering, Lanzhou Univ. of Tech., Lanzhou 730050, China
}

Wu Shiqiang, Zhou Hui , Xiao xiao \&Wang Yong

Nanjing Hydraulics Research Institute, Nanjing 210029, China

\begin{abstract}
The detailed experimental investigation on the 2D steady flow over a backward facing step in 30 Reynolds numbers range of $500<\mathrm{Re}<50000$ is carried out using particle image velocimetry ( PIV) and the separation and reattachment flow region over a backward facing step is displayed on a particle image. The characteristic of time-averaged flow field structure, the velocity distribution on the different sections along flow direction and vertical direction, and the change law of the length of separation and reattachment region and the location of vortex center are obtained from low Reynolds number $(\mathrm{Re}=506)$ to high Reynolds number $(\operatorname{Re}=47901)$.
\end{abstract}

KEYWORD: PIV; a backward facing step; high Reynolds number; the separation and reattachment flow region; hydraulic characteristics

\section{INTRODUCTION}

Backward step facing flow is one of common and complicated phenomenon in engineering, which has been used widely in water conservancy, energy, power, chemical, environmental protection and other departments. Backward step facing flow will produce lots of complicated hydraulic characteristics, involving the theoretical questions about the flow separation, the formation, development and evaluation of the vortex and the interaction between the vortex and the side wall. In engineering, how to utilize and control this phenomenon is very important, so the study of backward facing step flow characteristic is of great significant theoretical and practical value to engineering practice.

Many scholars home and abroad have conducted numerical modeling and experimental studies. In these studies, the researchers observed velocity distribution of different cross section in backward step facing flow by using hot-film (hot-wire) anemometer and laser velocimeter. They provide characters of the time-averaged flow fields of discrete section but they can't provide the transient and complete time-averaged flow fields. The spatial resolution of the flow images was low got by smokewire method and taking photos of the diesel sprays. Qi Er-rong, G. M. Di Cicca, Zhou jie, etc. get the flow characteristics of the 2-D backward facing step for Reynolds number $R e<6600$, utilizing particle image velocimetry (PIV) and the method of observation by means of segmentation to experiment about the separation and reattachment flow region over a backward facing step for Reynolds number $R e<6600$. Xiao xiao uses the 2-D k- $\varepsilon$ equations to simulate the backward step facing water flow, it is found the distribution of pressure behind the step and turbulent energy is also affected by the recirculation zone. The turbulent energy is less affected by the recirculation zone at low Reynolds numbers.

Although there are lots of researches about backward step facing flow at presents, they don't give a clear answer to the influence of Reynolds number and the shape of backward step on the separation and reattachment flow, and there are less researches on flow characteristics at high Reynolds number. The backward step facing flow in Reynolds number range of $500<\mathrm{Re}<50000$ is exhaustive observed and the hydraulic characteristics behind the step is discussed behind the step in the paper with large field of view particle image velocimetry (PIV), which can shows the complete separation and reattachment flow region over a backward facing step at an image.

\section{EXPERIMENT EQUIPMENT AND METHOD}

\subsection{Experiment equipment}

The experiment equipment is consist of water supply 
system, water tank, experimental channel, backwater system and is self-circulation system as shown in Fig 1 . It is made of $1.0 \mathrm{~cm}$ thick pmma and grey plastic plate with the thickness of $1.0 \mathrm{~cm}$. The channel is rectangular section, the step height $(h)$ is fixed at $h=2.2 \mathrm{~cm}$, the channel height $(H)$ in front of step is fixed at $H=6.6 \mathrm{~cm}$, the expansion $\operatorname{rate}(H / h)$ is equal to 3 . The depth of $\operatorname{channel}(B)$ is fixed at $B=20 \mathrm{~cm}$, the ratio of width over height $(B / h)$ is equal to 9.1. The distance between the channel import and step is $2.2 \mathrm{~m}$ and the distance between the channel export and step is $2.8 \mathrm{~m}$, which can make the flow of import and export develop fully.

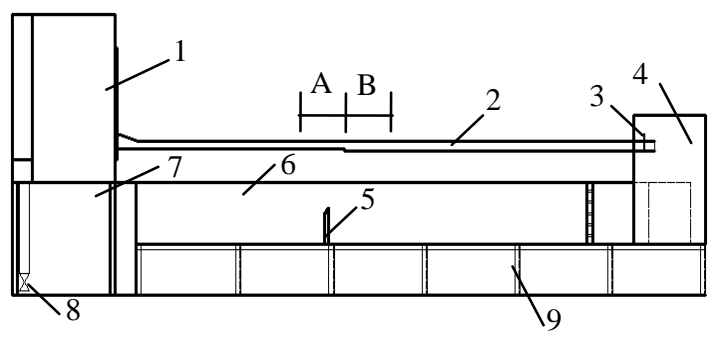

(a) Elevation drawing

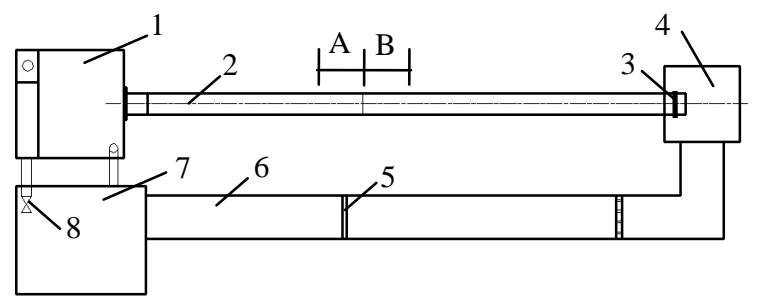

(b) Planar graph

1. water tank; 2. experimental channel; 3. End valve; 4. Stilling basin; 5. Measuring weir; 6. Return flume; 7. Pool; 8. Water pump; 9. support

Fig.1 Schematic diagram of experimental apparatus

\subsection{Measurement Method}

Using PIV system to measure backward step facing flow field on the symmetry plane of center channel, the tracer particle is glass beads, and its diameter is $1-5 \mu \mathrm{m}$, which can meet the following behaviors of water flow. This system mainly includes two subsystem; particle imaging system and particle images analysis system. The particle imaging system include the image resolution which is $4000 \times 2672$, which can shows the complete separation and reattachment flow region over a backward facing step at an image.

\subsection{Experiment method and content}

The definition of the Reynolds numbers of water flow is $R e=U_{0} h / v$, where $\mathrm{h}$ is the highness of step, $U_{0}$ is the average velocity of upstream channel which is in the front of steps, and $v$ is the kinetic viscosity. The coordinate system is shown in Fig. 2 with step vertex as the origin.

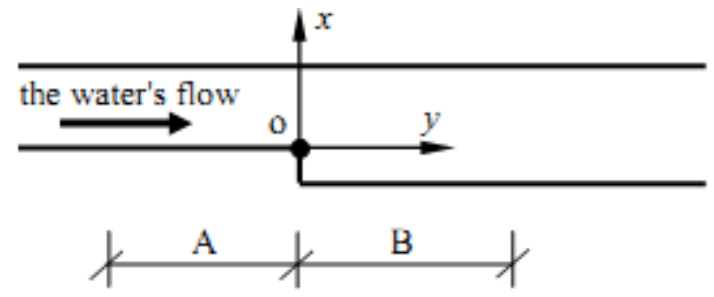

Fig.2 Schematic diagram of coordinate system

In the experiment, we measure the symmetry plane of section $A$ of the upstream channel and section B of the downstream channel as shown in Fig. 1. Each measuring length is $16 \mathrm{~h}$, the total length of measuring section is $32 \mathrm{~h}$. The flow field of the $2 \mathrm{D}$ steady flow over a backward facing step was measured in a Reynolds number range of $500<\mathrm{Re}<$ 50000 and obtained a total of 30 experimental groups Reynolds numbers. Two hundred and fortyeight flow-field images were measured in section A and $\mathrm{B}$ under the conditions of each the Reynolds number. The experiment includes laminar, transitive, and turbulent.

\section{EXPERIMENTAL RESULTS AND ANALYSIS}

Based on these data, the time-averaged velocity fields and the flow field distribution curves under some Re were gained by time-averaged method. The time-averaged velocity distributions in various states of flow were obtained by time-averaged method to the transient flow field of the same $R e$. The change law of the flow field structure, the velocity distributions, the length of the separation and reattachment flow region, and the location of vortex center are analyzed. Using the dimensionless numbers indicates the experimental results, and the dimensionless form of the position and velocity along the flowing direction(X) and along the height(Y) are $x / h, y / h, u / U_{0}, v / U_{0}$ respectively.

\subsection{Time-averaged flow field structure}

The time-averaged flow field diagram of the 2D steady flow over a backward facing step in different Reynolds number is shown in Fig.3. In different Reynolds number, the stable separation and reattachment region appears in a backward facing step, and the recirculation vortex in the recirculation region, which is in agreement with previous experimental conclusion. There is a second-time reflux phenomenon in the interior angle area of step when $R e$ is greater than 840 , and the length of the secondary circulation is longer when the Reynolds number is low. The length of the secondary circulation is between $1.7 \mathrm{~h}$ to $2.3 \mathrm{~h}$ in the case of 
$997 \leq R e \leq 1867$, and which is between $0.8 h$ to $1.3 h$ when $R e$ is greater than 1867 . The secondary circulation don't appear in the interior angle area of step when $R e$ is equals greater than 840 . The height of vortex ranges of $0.75 h \sim 0.85 h$, when $R e$ is equals less than 1495, which ranges of $0.85 h \sim 0.98 h$ in the case of $1495 \leq R e \leq 11237$, and which is considerable with the step height when $R e$ is equals greater than 11237.

Eaton and Johnston think, when the flow separates from the flow separation point, the flow immediately enter the turbulent state, and the length of the flow line is very short. But seeing from the chart of the flow structure obtained by the experiment, the length of the flow line is long when $R e$ is lower. The flow line substantially parallel with the wall in the range of $X \leq 2.5$ when $R e$ is equals less than 840, of which the length gradually reduce with increase of Reynolds number. The straight length of the flow line $(\mathrm{X})$ is $0.8 h$, which showed that the flow is a fully turbulent state after the straight flow line when $R e$ is greater than 4800 .

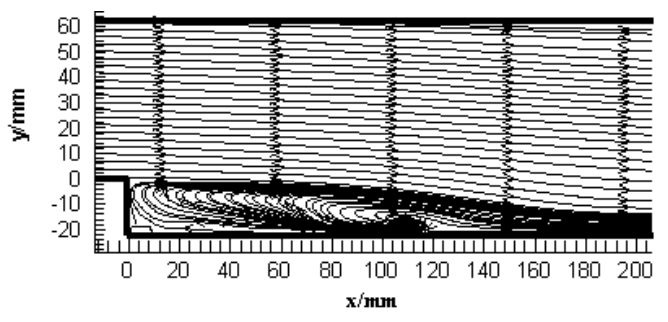

(a) $\mathrm{Re}=506$

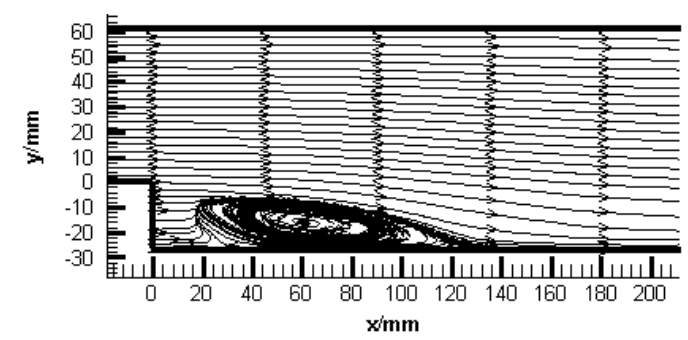

(b) $\mathrm{Re}=4800$

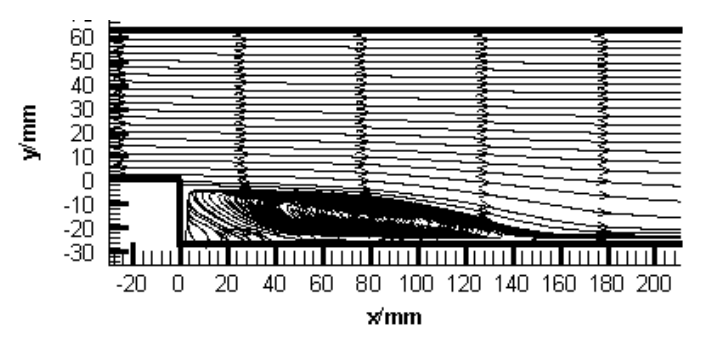

(d) $\mathrm{Re}=47901$

Fig. 3 The Time-averaged flow field structure diagram

\subsection{Velocity distribution}

The different velocity distribution in different Reynolds numbers at the different section $(x / h=-$ $1,1,3,5,7,9)$ is given in Fig. 4 . Near the step $(x / h=$ $1,3)$, the change of step boundary don't cause the sudden change of main flow velocity, but the main flow velocity of inflow shift down. With increasing distance from the step $(x / h=5,7,9)$, the main flow velocity becomes smaller, the maximum velocity gradually approaches the vertical $\operatorname{position}(y / h=1)$ until the flow is fully developed.

In the $\operatorname{section}(x / h=1,3)$ which is near step, velocity gradient which is near height of step ($0.3<y / h<0.5)$ is large. The velocity gradient is relatively small in the case of $\mathrm{y} / \mathrm{h}<0.3$ or $0.5<\mathrm{y} / \mathrm{h}$. With the increase of distance to the step $(x / h=5,7,9)$, the velocity gradient values becomes smaller, and of which the change is small under the location $(y / h<0.5)$. This indicates the water flow is affected by the sudden change of step boundary and produce shear flow the height section of near step, and the velocity gradient values is larger in the shear layer. The shear layer curves to wall surface, and the separated boundary layer after the reattachment redevelops with the increase of distance to the step.

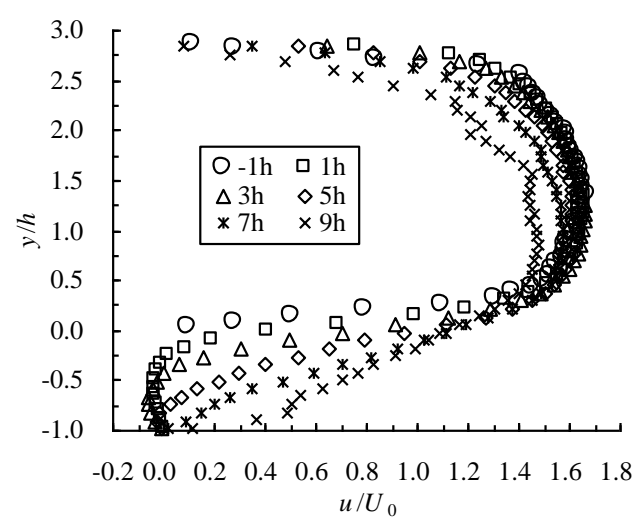

(a) $\operatorname{Re}=506$

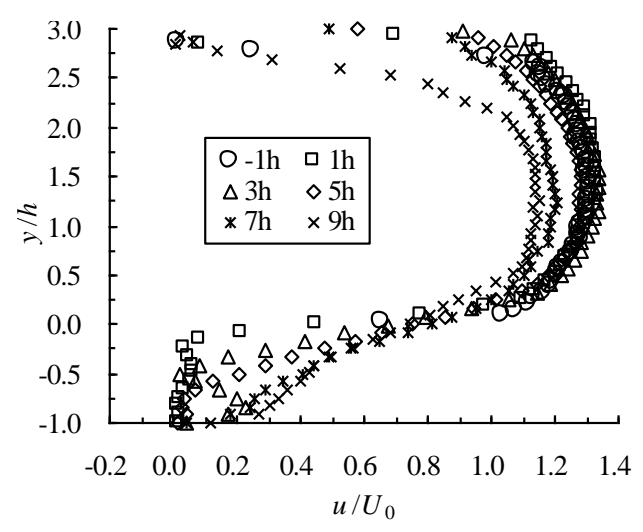

(b) $\mathrm{Re}=4800$

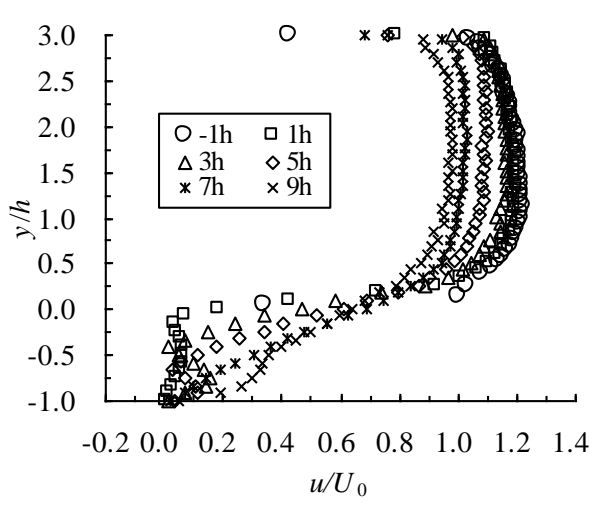

(c) $\mathrm{Re}=26160$ 


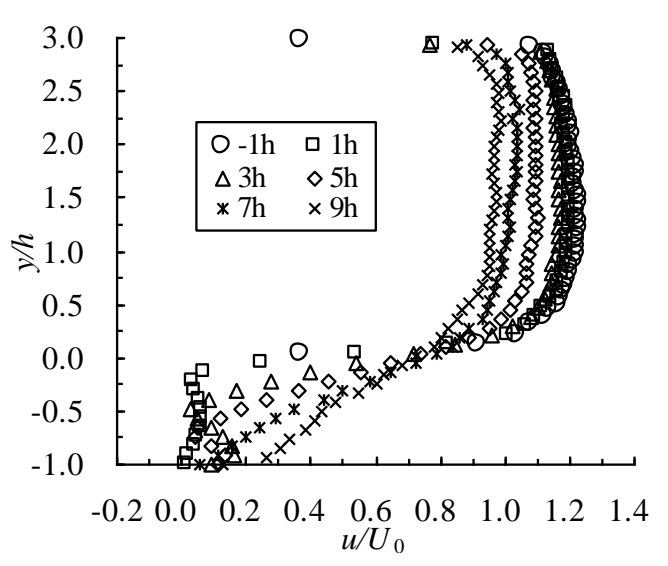

(d) $\mathrm{Re}=47901$

Fig. 4 The time-average velocity distribution diagram

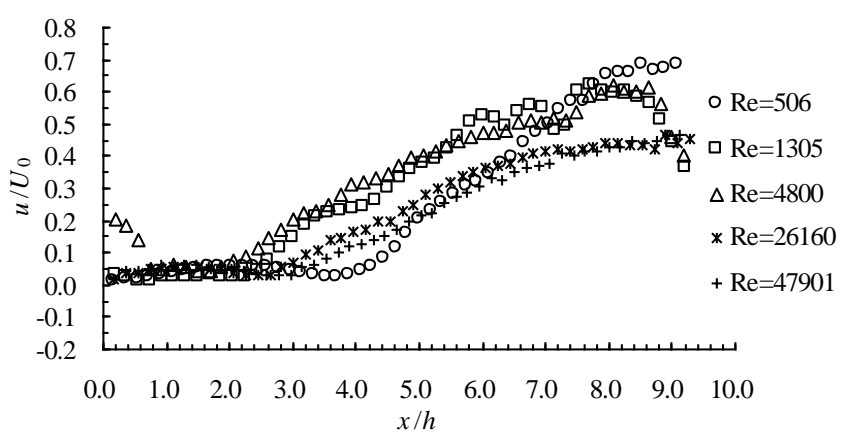

Fig. 5 The time-average velocity distribution of $1 / 2$ step height cross-section diagram

In the flow field of the backward-facing step, the change of characteristic parameter of different height section of step represent the flow field characteristic and reflect the change of turbulence intensity in separation and reattachment area. The time-averaged velocity distribution in $1 / 2$ step height cross-section of different Reynolds number is shown in Fig.5. As shown in Fig.5, the velocity increases slowly from the start of step, and its vertex appears at $x / h=1.29$, i.e. $\mathrm{Re}=47901$. With the increase of $\mathrm{x} / \mathrm{h}$, the velocity decrease slowly, and its lowest point appears and $u / U_{0}$ equal to 0.3 at $x / h=2.78$, i.e. $\operatorname{Re}=47901$. The velocity rapidly increase with the increase of $x / h$, and tends to be stable.

\subsection{Length of the separation and reattachment flow region}

The region flowing over a backward facing step is affected by sudden change of step boundary and boundary layer separates in step angle point, and the separation and reattachment area is formed in step downstream. This area contains an important feature of separation and reattachment flow, and there are several variations of the vortex, which indicates the location of the separation and reattachment point. The length of the separation and reattachment zone reflects one important characteristic quantity of timeaveraged flow over a backward facing step.
The change curve of length of the separation and reattachment zone in different Reynolds number is given in Fig.6. As shown in Fig.6, the length of the separation and reattachment zone $\left(X=x_{r} / h\right)$

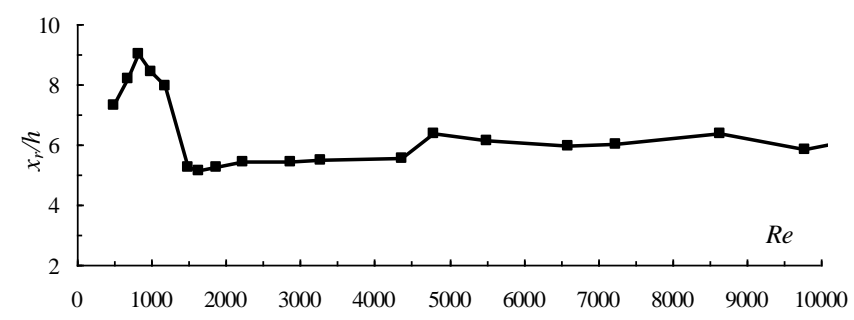

(a) $\operatorname{Re}=0 \sim 10000$

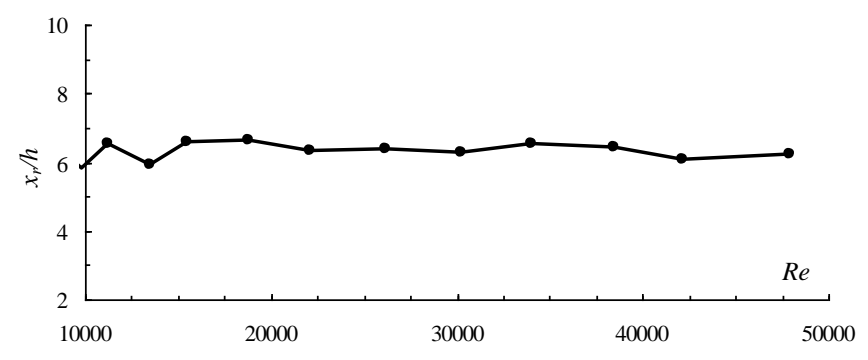

(b) $R e=10000 \sim 50000$

Fig. 6 The relation curve between the length of recirculation region and $R e$

increases with the increase of $\operatorname{Re}$ when $R e$ is less than 840, and the maximum value $(X=9.1)$ occurs in $R e=840$. The length $\left(X=x_{r} / h\right)$ decreases with the increase of $R e$ in the case of $840<R e \leq 1642$, a clear inflection point appears in $R e=1495$, and the minimum value $(X=5.1)$ occurs in $\mathrm{Re}=1642$. The length $\left(X=x_{r} / h\right)$ increases slowly with the increase of $R e$ in the case of $1642<R e \leq 4800$. The length $(X)$ maintains at $X=5.6 \sim 6.3$ in the case of $4800<\operatorname{Re} \leq 15523$. The length $(X)$ tends to be stable, its value is equal to 6.4 , and the flow has become a fully turbulent flow now.

\subsection{Location of vortex center}

The relation curve between vortex center position of the time-average flow field over a backward facing step and Re is shown in Fig. 7. As shown in Fig.7, the distance $(X)$ between Vortex center along the direction to separation point decreases with the increase of $R e$ When $R e$ is equals less than 4800, and its $\operatorname{value}(X)$ is in 2.6 5. The value $(X)$ increases slightly with the increase of $\mathrm{Re}$ in the case of $4800<R e \leqslant 15523$, and its value $(\mathrm{X})$ is in 3.0 3.5. The vortex center position along the vertical small changes. The vortex center position $(Y=y / h)$ along the vertical is approximately equal to 0.71 when $\mathrm{Re}$ is equals less than 4800 , and $\mathrm{Y}$ is approximately equal to 0.62 in the case of $4800<R e \leqslant 15523$. 


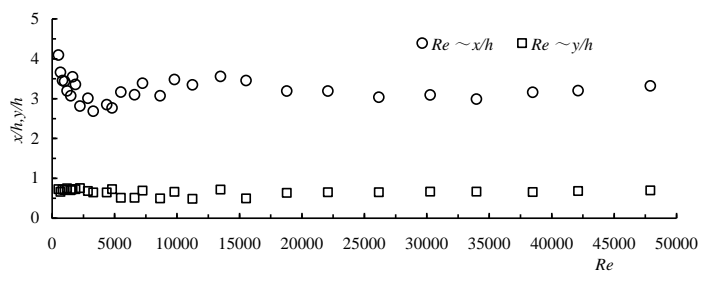

Fig.7 The relation curve between vortex center position and Re

\section{CONCLUSIONS}

Using a particle image velocimetry ( PIV), the detailed experimental investigation on the 2D steady flow over a backward facing step in 30 Reynolds numbers range of $500<\operatorname{Re}<50000$ is carried out and the separation and reattachment flow region over a backward facing step is displayed on a particle image. The characteristic of time-averaged flow field structure, the velocity distribution on the different sections along flow direction at $1 / 2$ height section and vertical direction, and the change law of the length of separation and reattachment region and the location of vortex center are obtained from low Reynolds number $(\mathrm{Re}=506)$ to high Reynolds number $(\operatorname{Re}=47901)$.

\section{ACKNOWLEDGEMENTS}

The National Natural Science Foundation (51379128) The National Natural Science Foundation (51269009)

\section{REFERENCES}

[1] CASTRO I P, BRADSHAW P. The turbulence structure of highly curved mixing layer. J Fluid Mesh, 1976, 73: 265304.

[2] SINLA S N, CUPA A K, OBERAI M M. Laminar separating flow over back steps and cavities (Part1 :back steps). AIAAJ, 1981, 12: 1527-1530.

[3] ARMALYB F, DURST F, PEREIRA J C F, et al. Experimental and theoretical investigation of backward facing step flow. J Fluid Mesh, 1983, 127: 473-496.

[4] Wang Jinjun, Lian Qixiang. Turbulent boundary layer separation reattachment and redevelopment over a backward facing srep. Acta Aeronautica Et Astronautica Ainica, 1994, 15(4): 393-398.

[5] Qi E R, Huang M H, Li W et. . Investigation of vortex st ructure of the $2 \mathrm{D}$ back ward facing step flow via PIV. Journal of Hydrodynamics, Ser A , 2004,19 (4) : 525 532

[6] G. M. Di Cicca, P. G. Spazzini, et al. PIV Investigation on a Controlled Backward Facing Step Flow. J Fluid Mesh, 2004, 6: 0-10.

[7] Zhou Jie1, Zeng Cheng, Wang Lingling. Numerical Simulation of Start Flow on Backward-facing Step with Free Surface. Water Power, 2008, 34(11): 27-30.

[8] Eaton, J, K. and Johnston, J, P., A Review of Research on Subsonic Turbulent Flow Reattachment, AIAA J., Vol. 19, pp. 1093-1100, sept. 1981

[9] Xiao Xiao, Fan Xin-jian, Wu Shi-qiang. Hydraulic characteristics of backward-facing step flow based on k- $\varepsilon$ turbulence model. Hydro-Science and Engineering, 2013, No.3: 25-31. 